\title{
Fatores que influenciam o acesso aos serviços de saúde na visão de profissionais e usuários de uma unidade básica de referência'
}

Factors associated to the access to health services from the
point of view of professionals and users of basic reference unit

\author{
Anna Paula Bise Viegas \\ Fundação Oswaldo Cruz. Centro de Pesquisas René Rachou. Labo- \\ ratório de Pesquisas Clínicas. Belo Horizonte, MG, Brasil. \\ E-mail: annapbvœgmail.com \\ Rose Ferraz Carmo \\ Escola de Saúde Pública do Estado de Minas Gerais. Belo Horizonte, \\ MG, Brasil. \\ E-mail: rferrazcarmo®gmail.com \\ Zélia Maria Profeta da Luz \\ Fundação Oswaldo Cruz. Centro de Pesquisas René Rachou. Labo- \\ ratório de Pesquisas Clínicas. Belo Horizonte, MG, Brasil. \\ E-mail: profetaळcpqrr.fiocruz.br
}

\section{Correspondência}

Zélia Maria Profeta da Luz

Centro de Pesquisas René Rachou. Fundação Oswaldo Cruz.

Av. Augusto de Lima, 1715, Barro Preto.

CEP 30190-002. Belo Horizonte, MG, Brasil.

\section{Resumo}

O objetivo deste trabalho foi analisar o acesso de usuários de uma Unidade Básica de Referência (UBR) aos serviços de saúde de um município da região Metropolitana de Belo Horizonte, Minas Gerais. Foram realizadas entrevistas semiestruturadas com 15 profissionais de saúde e aplicado questionário semiestruturado a 38 usuários. As entrevistas abordaram características do atendimento local, da equipe de saúde, dos usuários e da rede prestadora de serviços de saúde. Os questionários abordaram características sociodemográficas e de saúde dos usuários. A análise dos resultados das entrevistas e questionários foi apresentada com base nas dimensões estrutural (financiamento, cobertura), relacional (compreensão do processo saúde-doença) e operacional (organização dos serviços). Na dimensão estrutural, verificou-se que o subfinanciamento do sistema de saúde constitui uma dificuldade para o acesso aos serviços de saúde e para a implantação da Estratégia Saúde da Família (ESF); na dimensão relacional, a ausência de alinhamento sobre a estrutura e os fluxos de funcionamento do sistema de saúde impacta negativamente na relação do profissional com o usuário; e na dimensão operacional, profissionais de saúde e usuários relataram importantes lacunas tais como cobertura da ESF, marcação de consulta, acolhimento, barreiras geográficas, referência e contrarreferência. As informações obtidas neste trabalho são importantes para compreender os fatores que influenciam o acesso aos serviços de saúde da população estudada e colaborar para o desenvolvimento de estratégias voltadas para melhoria do acesso.

Palavras-chave: Acesso aos Serviços de Saúde; Assistência à Saúde; Políticas Públicas de Saúde.

1 Trabalho financiado pela Fundação Oswaldo Cruz (Fiocruz), Edital CSDT/Fiocruz - 001/2009. 


\section{Abstract}

This study aims to analyze the users' access to health services in a Basic Reference Unit (UBR) in a municipality of the metropolitan area of Belo Horizonte, Minas Gerais. The semi-structured interviews and questionnaire were conducted with 15 health professionals and 38 users respectively. The interviews addressed the local service, the health team, the users and the health services delivery network's characteristics. The questionnaire addressed users' sociodemographic and health characteristics. The interviews and questionnaires results analysis were presented on structural (financing, coverage), relational (comprehension of the health-illness process) and operational (service organization) dimensions. In the structural dimension, it was observed that the health system underfunding is a difficulty to the health services access and to the Family Health Strategy (ESF) implementation. In the relational dimension, it was observed that the lack of alignment regarding the structure and the health system operational flow impact negatively on the relationship between health professional and user. On the operational dimension, the health professionals and users reported significant shortcomings, such as the ESF coverage, appointment scheduling, reception, geographical barriers, referral and counter referral. The information obtained in this study is important to understand the factors associated to the health services access for the subject population and to help the developing strategies to improve the access. Keywords: Health Services Accessibility; Delivery of Health Care; Public Health Policies.

\section{Introdução}

Mais de 20 anos após a implementação do Sistema Único de Saúde (SUS) e, apesar de avanços relacionados, sobretudo, à ampliação da oferta de serviços da rede básica de saúde, o acesso aos serviços de saúde ainda constitui um desafio. Efetivar o direito constitucional ao acesso universal e integral envolve determinantes inúmeros e complexos aos quais diferentes estudiosos tem se debruçado (Thiede; McIntyre, 2008; Donabedian, 2003; Goddard; Smith, 2001; Travassos; Martins, 2004; Andersen, 1995; Penchansky; Thomas, 1981; Donabedian, 1973). A própria definição do termo acessibilidade conta com diferentes abordagens: Frenk (1985 apud Travassos; Martins, 2004, p. 193) utiliza esse termo abordando características da oferta e da população. A acessibilidade seria influenciada pelo fator "resistência" (obstáculos ecológicos, financeiros e organizacionais para procurar e obter cuidados) e pelo "poder de utilização" (capacidade da população para superar tais obstáculos).

Para Andersen (1995), o acesso seria influenciado pela predisposição do indivíduo para utilização do serviço de saúde (características demográficas, estrutura social e crenças), por fatores capacitantes que permitem e que impedem a utilização (renda, seguro de saúde, disponibilidade de serviços, distância e tempo de espera) e pela necessidade de saúde do indivíduo.

Segundo Donabedian (1973, 2003), o conceito de acessibilidade refere-se à facilidade com que as pessoas obtêm assistência à saúde. Para o autor, a acessibilidade depende de fatores sócio-organizacionais e geográficos que se relacionam. $\mathrm{O}$ autor ainda cita os fatores sociais e culturais e as preferências étnicas e religiosas como importantes para a acessibilidade e, embora enfatize fatores geográficos e organizacionais, considera a acessibilidade como um importante fator da aceitabilidade social para a assistência.

Para Thiede e McIntyre (2008), o conceito de acesso é abordado como liberdade para uso, baseada na consciência da possibilidade de uso pelo indivíduo e no seu empoderamento para escolha. Esses autores relacionam fatores como a existência de um serviço específico ao alcance do indivíduo, bem como cordialidade com o usuário; a existência de 
sistemas de marcação e conveniência de horários; a capacidade do paciente em arcar com os custos diretos e indiretos da assistência e o modelo de financiamento do sistema de saúde; e os fatores subjetivos, sociais e culturais. Dessa forma, o nível de acesso aos serviços de saúde seria determinado pelo grau de adequação entre indivíduos e comunidades e o sistema de saúde.

Segundo Obrist et al. (2007), o acesso aos serviços de saúde pode ser determinado por situações de vulnerabilidade da população, que depende do capital humano, físico, financeiro e social.

As contribuições de diferentes estudiosos do acesso aos serviços de saúde sinalizam a complexidade do tema e sua relação com a percepção das necessidades de saúde, para a conversão dessas necessidades em demandas e destas em uso. Nesse contexto, é pertinente compreender os fatores que influenciam a utilização dos serviços de saúde identificando razões para possíveis diferenças na procura, na satisfação com o cuidado recebido e nos resultados alcançados. Baseado nesses pressupostos, este estudo buscou, a partir da perspectiva de profissionais de saúde e de usuários de uma Unidade Básica de Referência, identificar fatores que influenciam o acesso aos serviços públicos de saúde pela população em um município de Minas Gerais.

\section{Métodos}

\section{Local do estudo}

O estudo foi realizado em uma localidade com população estimada de 40.000 habitantes, área de abrangência de uma unidade de saúde, em município da Região Metropolitana de Belo Horizonte (RMBH).

Em relação aos serviços de saúde, a localidade conta com 12 Unidades Básicas de Saúde (UBS) e uma Unidade Básica de Referência (UBR). Aproximadamente cinco mil famílias são cadastradas na Estratégia Saúde da Família (ESF), distribuídas nas UBS. A UBR é responsável pelo atendimento referenciado das unidades da ESF nas clínicas básicas (Clínica Médica, Pediatria e Ginecologia), no atendimento odontológico e no de Enfermagem. Eventualmente, a UBR oferece atendimento em clínicas especializadas, como em Pneumologia e Psiquiatria.

Para o cadastro da UBR, são utilizadas as in- formações da ESF dos moradores da área adscrita. A parcela da população que não tem acesso a ESF pode ser atendida na UBR, desde que possua uma declaração de que não é cadastrada, que pode ser emitida em unidade da ESF.

A UBR onde foi desenvolvido o estudo conta com 39 profissionais: 12 médicos, uma psicóloga, dois dentistas, quatro enfermeiras, dez auxiliares de enfermagem, dois auxiliares de consultório dentário (ACDs), e oito auxiliares relacionados à administração da Unidade.

A unidade funciona de segunda a sexta-feira, das 7 às 19 horas. Por mês, são realizadas aproximadamente 1.070 consultas sendo $230(21,5 \%)$ de Pediatria, 580 (54,2\%) de Clínica Médica e 260 $(24,3 \%)$ de Ginecologia.

\section{Sujeitos da pesquisa e coleta das informações}

Os sujeitos da pesquisa foram profissionais de saúde do nível central da Secretaria Municipal de Saúde (SMS) e da UBR - gestores, profissionais assistenciais de nível superior e de nível médio - e usuários da UBR.

Para a coleta das informações com os profissionais, foram realizadas, em fevereiro e março de 2011, entrevistas semiestruturadas guiadas por roteiros previamente elaborados que abordavam características do atendimento local, da equipe de saúde, dos usuários e da rede prestadora de serviços de saúde.

Os profissionais que participaram do estudo foram identificados como gestor 1, gestor 2, profissional de nível superior 1, profissional de nível médio 1 , etc.

Para a pesquisa com os usuários, foram utilizados questionários semiestruturados aplicados nos meses de março e abril de 2011. A amostragem não pôde ser aleatória, já que o cadastro da população usuária da UBR é diferenciado - parte da população possui prontuário familiar (cadastro da ESF) e parte possui prontuário individual (população não cadastrada na ESF) - impossibilitando o sorteio. Optou-se pela amostragem não probabilística e por conveniência, entrevistando os usuários que estavam na unidade nos dias da entrevista e que aceitaram participar do projeto. Os usuários foram entrevistados sempre às sextas-feiras, o que pode ter selecionado um grupo específico de indivíduos. 
Os entrevistadores abordavam os usuários que estavam aguardando atendimento, explicavam o teor da pesquisa e faziam o convite para participação no estudo. Os questionários eram anônimos e os critérios de inclusão dos usuários foram indivíduos com 18 anos ou mais, de ambos os sexos e usuários da UBR.

O questionário foi dividido em quatro blocos que contemplavam as características dos usuários; da moradia; informação e comunicação; e de saúde dos usuários (morbidade; cobertura de plano de saúde; acesso aos serviços de saúde; utilização dos serviços de saúde). O questionário foi organizado em 68 questões, sendo 23 fechadas, 25 abertas e 20 mistas. Os entrevistadores foram responsáveis pelo preenchimento das respostas, que foi feito rigorosamente de acordo com a resposta do entrevistado.

Os depoimentos dos profissionais foram gravados, integralmente transcritos e tratados pela análise de conteúdo temática (Minayo; Deslandes; Gomes, 2010; Bardin, 2010). Tal técnica viabiliza a análise do conjunto e das particularidades e a classificação das informações em categorias (Bardin, 2010).

\section{Análise das informações}

Para a análise dos questionários foi construído um banco de dados no Programa EpiInfo, do qual se procedeu à análise descritiva dos dados obtidos.

Foi realizada sistematização teórica das condições de acesso da população estudada, adotando as dimensões "estrutural”, "operacional” e "relacional”.
Essas dimensões remetem, de alguma forma, à abordagem teórica que Giovanella e Fleury (1996) adotam para explicar as dimensões do acesso (política, econômica, técnica e simbólica), além de retratarem a acessibilidade, discutida por Donabedian (1973, 2003), como diretamente associada a fatores sócio-organizacionais e geográficos e a questão da subjetividade no acesso aos serviços de saúde, aprofundada por Thiede e McIntyre (2008) que abordam o conceito como liberdade para uso, baseada na consciência da possibilidade de uso pelo indivíduo e no seu empoderamento para escolha.

A dimensão "estrutural" seria a mais distal e relacionada a fatores gerais, como o contexto socioeconômico do município que impacta não apenas o acesso, mas de forma mais abrangente as condições de vida da população. A dimensão "operacional” ocupa uma posição intermediária entre as dimensões "estrutural" e "relacional" (Figura 1) e, na narrativa dos sujeitos da pesquisa, englobou toda gama de elementos relacionados com a organização do serviço de saúde, perpassando todos os níveis de atenção e suas particularidades. A dimensão "relacional” abarca a compreensão do processo saúde-doença e todos elementos culturais envolvidos nessa compreensão: percepções, crenças, valores, ou como expresso por Canguilhem (2000) os "modos de andar a vida" que colocam fatores associados à saúde em campos de normatividade situados além dos limites do corpo físico, orientados às suas condições de expressão na vida cotidiana.

\section{Figura I - Dimensões teóricas usadas para análise do acesso aos serviços de saúde na região estudada e cate- gorias empíricas identificadas a partir da fala dos sujeitos}

Dimensões teóricas

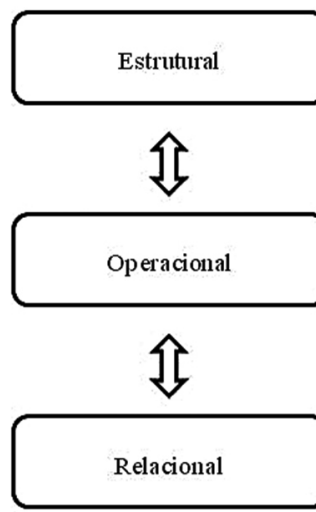

Categorias empiricas

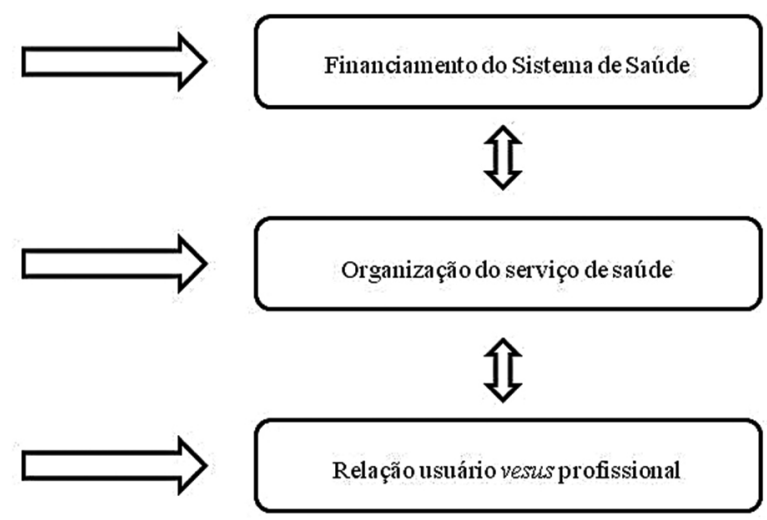


O projeto foi aprovado pelo Comitê de Ética em Pesquisa com Seres Humanos do Centro de Pesquisas René Rachou - CPqRR/Fundação Oswaldo Cruz, em 21 de junho de 2010, por meio da Carta de Aprovação n ${ }^{0}$ 17/2010, protocolo CEP - CPqRR n ${ }^{0}$ 16/2010, CAAE 0015.0.245.000-1.

\section{Resultados e discussão}

Foram entrevistados 15 profissionais de saúde, entre trabalhadores do nível central da SMS (2) e da UBR (13), sendo sete $(46,7 \%)$ de nível médio e oito $(53,3 \%)$ de nível superior. Três (20\%) dos profissionais trabalhavam na gestão; os demais trabalhavam na área assistencial. O tempo de atuação no cargo e na UBR foi variável, com medianas de 5,5 e 3,0 anos, com intervalos entre valores mínimos e máximos de o,6 a 25 anos e o a 12 anos, respectivamente (Tabela 1).

Tabela I - Número e proporção (\%) de profissionais de saúde da Secretaria Municipal de Saúde e Unidade Básica de referência entrevistados segundo escolaridade e atuação em um município da Região Metropolitana de Belo Horizonte, 2011

\begin{tabular}{lcc} 
Perfil dos Profissionais entrevistados & $n$ & $\%$ \\
Escolaridade & 15 & 100 \\
Nível Médio & 7 & 46,7 \\
Nível Superior & 8 & 53,3 \\
Atuação & 15 & 100 \\
Gestão & 3 & 20 \\
Assistência & 12 & 80 \\
Tempo (anos) & Mediana & Mín-máx \\
Atuação no cargo & 5,5 & $0,6-25,0$ \\
Trabalho na UBR & 3 & $0,0-12,0$ \\
\hline
\end{tabular}

Na análise dos questionários, foram identificados, também, aspectos semelhantes às categorias observadas na análise de conteúdo das falas dos profissionais. Para possibilitar o diálogo entre as informações, os resultados foram apresentados de acordo com esta categorização.

\section{Usuários}

Foram entrevistados 38 usuários da UBR. Aproximadamente $50 \%$ deles tinha até 40 anos de idade. As idades variaram de 18 a 6 o anos em 31 (81,6\%) usuários e sete $(18,4 \%)$ com idades superiores a 60 anos. Entre os 38 usuários, houve predomínio do sexo feminino, com $24(63,2 \%)$ mulheres. Dos 38 usuários, seis $(15,8 \%)$ nunca estudaram ou foram apenas alfabetizados. A renda familiar de 19 (65,4\%) dos entrevistados era inferior a três salários mínimos e 27 (71\%) viviam há 10 ou mais anos na região (Tabela 2).

Tabela 2 - Número e proporção (\%) dos usuários da Unidade Básica de Referência entrevistados segundo variáveis sócio-demográficas em um município da Região Metropolitana de Belo Horizonte, 2011.

\begin{tabular}{|c|c|c|}
\hline Dado sociodemográfico & $n$ & $\%$ \\
\hline Faixa etária & 38 & 100 \\
\hline 18 a 30 anos & 12 & 31,6 \\
\hline 31 a 40 anos & 6 & 15,8 \\
\hline 41 a 50 anos & 6 & 15,8 \\
\hline 51 a 60 anos & 7 & 18,4 \\
\hline$>60$ anos & 7 & 18,4 \\
\hline Sexo & 38 & 100 \\
\hline Masculino & 14 & 36,8 \\
\hline Feminino & 24 & 63,2 \\
\hline Escolaridade & 38 & 100 \\
\hline Até ensino fundamental & 18 & 47,4 \\
\hline Até ensino médio & 11 & 29 \\
\hline Até ensino superior & 3 & 7,9 \\
\hline Não estudou/ alfabetização & 6 & 15,8 \\
\hline Renda familiar em salários mínimos* & $29^{* *}$ & 100 \\
\hline I a 1,9 & 13 & 44,8 \\
\hline 2 a 2,9 & 6 & 20,6 \\
\hline 3 a 4,9 & 8 & 27,5 \\
\hline 5 a 8 & 2 & 6,8 \\
\hline Tempo de moradia na região & 38 & 100 \\
\hline Menos de 10 anos & II & 29 \\
\hline 10 a 29 anos & 18 & 47,2 \\
\hline 30 ou mais anos & 9 & 23,7 \\
\hline
\end{tabular}

*Valor de referência do salário mínimo (2011): $R \$ 545,00$

**Dados válidos 


\section{Fatores relacionados ao acesso aos serviços de saúde}

Buscando articular a dimensão teórica com a narrativa dos sujeitos da pesquisa, foram identificadas as categorias empíricas: "financiamento do sistema de saúde"; "organização do serviço de saúde”; e "relação usuário versus profissional” (Figura 1).

Algumas percepções/opiniões sobre o acesso aos serviços de saúde - como esperado - não foram consensuais entre os sujeitos da pesquisa (usuários e profissionais). No decorrer da apresentação dos resultados, os consensos e dissensos ficam nítidos. Ressaltamos que o objetivo da inclusão desses atores sociais foi contribuir com a compreensão dos fatores que influenciam o acesso aos serviços de saúde na região estudada.

\section{Dimensão estrutural}

O principal elemento da dimensão "estrutural" identificado na narrativa dos sujeitos da pesquisa foi o financiamento do sistema de saúde, que, no município, constitui uma importante lacuna para o acesso aos serviços de saúde. Para exemplificar, basta analisar a despesa total com cada habitante do município que, em 2009, foi equivalente a $\mathrm{R} \$ 187,72$, sendo $\mathrm{R} \$$ 76,05 recursos próprios ${ }^{2}$, e comparar com a média dessa despesa no Brasil, no ano de 2004, cinco anos antes, que totalizou R\$359,0o, sendo R\$ 9o,oo de recursos municipais (RIPSA, 2011). Como a grande parte da população do município é pobre, a arrecadação de impostos é muito pequena. Segundo um dos gestores entrevistados, a arrecadação de impostos não é suficiente: [...] recursos o município não tem. Hoje, somente $20 \%$ das pessoas pagam o seu IPTU [...] (Gestor 2).

Embora o governo municipal invista $31,4 \%$ de seus recursos em saúde ${ }^{3}$, - mais que o dobro dos $15 \%$ exigido pela Emenda Constitucional 29 de 2000 (Brasil, 200o), esses recursos ainda não são suficientes para os investimentos e custeio necessários ao sistema de saúde local.

Um agravante do contexto local é o fato do município estudado conviver com o estigma da violência. Esse rótulo foi apontado nas entrevistas como um fator local de determinação do acesso, principalmente por estar associado ao déficit de recursos humanos:
[...] acho que é uma cidade muito estigmatizada [...] A violência que perpassa o imaginário de todo mundo [...] À medida que os profissionais vêm e percebem que não é esse horror todo, conseguem trazer outros, conseguem se fixar mais (gestor 1).

O financiamento do sistema de saúde, inserido na dimensão estrutural do acesso, ressaltou como a vulnerabilidade social e econômica de um município impacta negativamente o acesso de sua população aos serviços de saúde e reforça que mudanças na dimensão estrutural do acesso requerem, como apontado por Jesus e Assis (2010), o comprometimento dos formuladores de políticas com o acesso universal aos serviços de saúde; a pactuação entre instâncias de poder, governo, sociedade civil, prestadores de serviços públicos e/ou privados e instituições formadoras de recursos humanos em saúde; o acompanhamento do processo e da tomada de decisão partilhada entre os diferentes sujeitos com representação política e transparência no processo decisório.

\section{Dimensão operacional}

Foram relatadas importantes lacunas da entrada do indivíduo no serviço (cobertura da ESF, marcação de consulta, acolhimento, barreiras geográficas) até a resolução completa do "problema" (fluxo de atendimento: referência e contrarreferência).

Foi abordada pelos sujeitos da pesquisa a baixa cobertura da ESF, que está relacionada à limitação de recursos e a problemas organizativos da ESF no município. A rotatividade da população foi relatada por um profissional assistencial de nível superior, explicando que o cadastramento de famílias nas unidades da ESF não acompanha essa dinâmica, levando à permanência do cadastro de famílias que não moram mais na região, enquanto outras residentes na região não conseguem se cadastrar.

Essas questões foram confirmadas pelos usuários que relataram a demanda pelo cadastramento. Apenas 39,5\% dos entrevistados afirmaram ser cadastrados na ESF (Tabela 3): gostaria de ser cadastrada, mas o posto do bairro já está saturado. Não consigo ser atendida (usuário 37).

2 Informações do DATASUS consultadas no Cadernos de Informações de Saúde: Minas Gerais: Ribeirão das Neves, 2011. Disponível em: <http://tabnet.datasus.gov.br/tabdata/cadernos/mg.htm>. Acesso em: 7 dez. 2011.

3 Informações do DATASUS consultadas no Cadernos de Informações de Saúde de Minas Gerais e Ribeirão das Neves, em 2o11. Disponível em: <http://tabnet.datasus.gov.br/tabdata/cadernos/mg.htm>. Acesso em: 7 dez. 2011. 
Tabela 3 - Número e proporção (\%) dos usuários da Unidade Básica de Referência entrevistados segundo características do acesso aos serviços de saúde em um município da Região Metropolitana de Belo Horizonte, 2011

\begin{tabular}{|c|c|c|}
\hline Caracterização do acesso aos serviços de saúde & $n$ & $\%$ \\
\hline \multicolumn{3}{|l|}{ Cadastramento na Estratégia de Saúde da Família } \\
\hline Sim & 15 & 41,7 \\
\hline Não & 21 & 58,3 \\
\hline Total & $36^{*}$ & 100 \\
\hline \multicolumn{3}{|c|}{ Tempo gasto pelos participantes para ir de casa ao serviço do bairro ( $\mathrm{min}$. ) } \\
\hline$<10$ & 5 & 13,2 \\
\hline $10 \mathrm{a} 15$ & 19 & 50 \\
\hline 16 a 30 & 10 & 26,3 \\
\hline 31 a 60 & 3 & 7,9 \\
\hline$>60$ & 1 & 2,6 \\
\hline Total & 38 & 100 \\
\hline \multicolumn{3}{|c|}{ Motivos de utilização do serviço do bairro pelo participante do estudo } \\
\hline É perto & 18 & 53 \\
\hline É onde está cadastrado & 5 & 14,7 \\
\hline É gratuito & 3 & 8,8 \\
\hline O problema era simples & 6 & 17,6 \\
\hline Outros & 2 & 5,9 \\
\hline Total & $34^{*}$ & 100 \\
\hline \multicolumn{3}{|c|}{ Motivos do participante para utilizar serviços de saúde de fora do bairro } \\
\hline Não há vaga/médico no bairro & 13 & 34,2 \\
\hline Quando tem problema grave & 8 & 21,1 \\
\hline Não é cadastrado na ESF & 3 & 7,9 \\
\hline Atendimento ruim & 2 & 5,3 \\
\hline Outros & 9 & 23,7 \\
\hline Sem informação & 3 & 7,9 \\
\hline Total & 38 & 100 \\
\hline \multicolumn{3}{|c|}{ Opinião do participante do estudo sobre o serviço de saúde do bairro } \\
\hline Bom & 10 & 26,3 \\
\hline Mais ou menos & 9 & 23,7 \\
\hline Ruim & 9 & 23,7 \\
\hline Muito ruim & 8 & 21,1 \\
\hline Não opinou & 2 & 5,3 \\
\hline Total & 38 & 100 \\
\hline \multicolumn{3}{|c|}{ Principal difıculdade do participante para utilizar o serviço do bairro } \\
\hline Não há médicos & 14 & 36,8 \\
\hline Demora em marcar & 12 & 31,6 \\
\hline Atendimento ruim & 4 & 10,5 \\
\hline Mudança do médico & 2 & 5,3 \\
\hline Distância/dificuldade de locomoção & 2 & 5,3 \\
\hline Não há dificuldade & 3 & 7,9 \\
\hline Sem informação & 1 & 2,6 \\
\hline Total & 38 & 100 \\
\hline
\end{tabular}

* Dados válidos 
Com relação à marcação de consultas, gestores e profissionais assistenciais ressaltaram a existência de marcação livre e agenda aberta na UBR, entretanto, os depoimentos mostraram que esta "liberdade" é regulada, já que a marcação é feita para curtos períodos, na tentativa de evitar o absenteísmo: [...] fica um tempo muito longo (entre a marcação e a consulta) $e o$ paciente esquece essa consulta (profissional de nível superior 2). A falta e a rotatividade dos profissionais, sobretudo médicos, e o grande percentual da população que não é cadastrada na ESF foram abordados como fatores que colaboram para a dificuldade de marcação: [...] são poucos médicos e a demanda é muito grande (profissional de nível médio 5). Um fator positivo relatado foi não existir mais filas para marcação de consultas de madrugada.

No entanto, 6o, $5 \%$ (23) dos usuários entrevistados consideraram a forma de marcação de consultas ruim. Um usuário relata: $\varepsilon$ difícil marcar clínico, ginecologista e pediatra. Tem que vir de madrugada para pegar senha (usuário 5).

O tempo de espera entre marcação e atendimento não é longo, sendo de aproximadamente uma ou duas semanas, entretanto, foi relatada grande espera por exames complementares pelos usuários: $O$ município não fez convênio para raios-X. Fiquei dois meses esperando e tive que fazer particular (usuário 19).

No que diz respeito à marcação de consultas e exames da atenção secundária, foram abordadas nos depoimentos a grande demanda por atendimento especializado, que foi associada à pouca resolutividade dos médicos da APS, e a demora na marcação de consultas, associada à falta de especialistas e dificuldades na regulação do sistema de saúde municipal: [...] porque os clínicos e pediatras [...] que atendem no nível primário, eles poderiam ser mais resolutivos [...] $\varepsilon^{\varepsilon}$ muito simples um profissional receber um cliente e não procurar resolver o problema dele e rapidamente encaminhá-lo para especialista [...] (gestor 3).

A questão da marcação de consultas mostrou-se um importante determinante para o acesso dos moradores da região estudada, corroborando o encontrado por Cunha e Vieira-da-Silva (2010), em um estudo avaliativo sobre a acessibilidade aos serviços de saúde em um município baiano. Esses autores identificaram que a dificuldade para marcação de consultas e os longos tempos de espera para o atendimento foram uns dos aspectos que mais contribuíram para a baixa pontuação das unidades de ESF avaliadas. Os autores estabeleceram como critérios para uma marcação ideal a marcação diária, sem horário fixo para a demanda espontânea, por meio de grupos e demandas dos Agentes Comunitários de Saúde (ACS); a marcação de consulta por telefone; a priorização segundo critérios de gravidade/ necessidade por profissionais de nível superior; a marcação comunicada pelo ACS ou pela unidade; a existência de lista de espera e a substituição do paciente faltoso de modo sistemático; e o tempo de espera entre a marcação e o atendimento de até cinco dias. Observa-se que entre esses critérios, o único presente na unidade de saúde avaliada neste trabalho foi a inexistência de horário fixo para marcação, embora a marcação não seja diária, já que a agenda fica "fechada" periodicamente.

Em relação ao referenciamento, Pena et al. (2012), em seu estudo sobre a integralidade do cuidado ao paciente com doença renal, observou que metade das pessoas com a doença em estágio leve ou moderado eram encaminhadas ao nível especializado. Segundo os autores, o impacto nas agendas do nível especializado pela demanda por encaminhamento poderia acarretar em comprometimento da equidade devido ao estrangulamento no acesso dos casos avançados da doença ao especialista. Esse distanciamento implicaria, também, na possibilidade de retenção desses pacientes pelo nefrologista, sem a garantia da contrarreferência, rompendo com a lógica do vínculo terapêutico e comprometendo a qualidade do cuidado. Os autores sugerem o "apoio especializado matricial", numa lógica do cuidado paciente-centrado, como uma possível solução para a questão.

Foi possível identificar alguns contrastes sobre o acolhimento, como: acolhimento promovendo o acesso versus acolhimento proporcionando acesso muito fácil (alguns profissionais consideraram que a forma atual de funcionamento do sistema cria facilidades em excesso para o usuário, que, segundo os profissionais, acaba utilizando desnecessariamente os serviços); contrastes entre as necessidades dos usuários, suas demandas versus as ofertas de serviços de saúde/escuta pobre do usuário pelos 
profissionais de saúde (foi citada a dificuldade dos serviços e profissionais em acolher as reais necessidades dos usuários); cadastrados acolhidos versus não cadastrados não acolhidos (os indivíduos não cadastrados na ESF têm grande dificuldade para ter acesso aos serviços de saúde): [...] o acolhimento é muito bacana [...] Mas tem o lado negativo, porque dá um acesso muito fácil para eles (gestor 3); $\mathrm{Na}$ verdade, a gente sente que não há interesse de perguntar qual é o problema que trouxe o sujeito aqui (profissional de nível superior 5).

A acessibilidade geográfica foi abordada pelos profissionais de saúde que apontaram como fatores dificultadores do acesso a distância da residência do usuário à unidade, a deficiência do transporte público no município e a impossibilidade do pagamento da passagem de ônibus devido às dificuldades econômicas dos usuários. Nas entrevistas com os usuários, entretanto, observou-se que $63,2 \%$ gastavam menos de 15 minutos para chegar à UBR (Tabela 3) e 84,2\% iam a pé. Essa divergência reflete os usuários que mais frequentam a unidade: aqueles que residem em suas proximidades.

Esses fatores determinantes para a acessibilidade geográfica costumam ser relatados quando se avalia populações rurais (Travassos; Viacava, 2007; Pinheiro et al., 2002). Embora a área do estudo seja urbana, nota-se que esta urbanização é irregular e precária e a região não é atendida por sistema de transporte adequado. Travassos e Viacava (2007) discutem que a distância dos serviços às residências dos usuários pode ser compensada por medidas como a facilitação de transporte, a presença de sistema de referência aos serviços hospitalares e mecanismos de acolhimento que facilitem a obtenção de cuidado.

Os sujeitos da pesquisa relataram problemas no fluxo de atendimento, sendo que os usuários destacaram, sobretudo, fatores associados à referência, como a marcação de consultas, apresentada acima. Os profissionais de saúde, por sua vez, destacaram aspectos relacionados à contrarreferência, associando-os à formação dos profissionais da atenção secundária e terciária: [...] esse já é um trabalho mais de formação [...] Não se sabe o porquê dos profissionais das especialidades não terem interesse de contrarreferenciar (gestor 3 ).
A questão da referência e contrarreferência mostra-se um problema recorrente em todo o país. Cunha e Vieira-da-Silva (2010), em seu estudo sobre acesso aos serviços de saúde em um município da Bahia, evidenciaram que o sistema de referência e contrarreferência foi um dos fatores de determinação do acesso da população que mais mostrou deficiências. Moimaz et al. (2010), em um estudo conduzido com usuários em cinco municípios de São Paulo, mostraram que a deficiência no sistema de referência e contrarreferência constitui um problema no acesso aos serviços de saúde e, consequentemente, dificulta o atendimento das necessidades da população, diminuindo sua satisfação em relação aos serviços prestados.

Parece-nos, particularmente importante, ressaltar que a dimensão "operacional” configura ainda um importante obstáculo para a efetiva consolidação do SUS e de seus princípios. Concordamos nesse ponto com a proposição de Santos e Andrade (2012) de que ainda há um vasto espaço entre as necessidades de saúde da população e a rede pública de ações e serviços de saúde. Esses autores destacam que os processos organizativos do SUS, até o presente momento, tateiam entre a descentralização que fragmenta os serviços e a regionalização que deve uni-los em rede integrada e referenciada capaz de dar respostas objetivas aos problemas demandados pelos usuários. Para tanto, como nos aponta Jesus e Assis (2010) é necessário fortalecer a porta de entrada do sistema de saúde, demarcando os fluxos dos atendimentos organizados a partir das demandas epidemiológica, sanitária e social.

\section{Dimensão relacional}

Na dimensão relacional, observou-se que a ausência de alinhamento referente à estrutura e aos fluxos de funcionamento do sistema/serviço de saúde, impacta negativamente na relação do profissional com o usuário. Andrulis (200o), em seu trabalho que discute estratégias para melhorar o acesso aos serviços de saúde nos centros urbanos, aponta a questão da dificuldade de compreensão do processo saúde-doença e do funcionamento do sistema de saúde por uma parcela da população (especialmente idosos, pobres e alguns grupos culturais) como um fator que prejudica a inserção do cidadão no sistema e no 
cuidado à sua saúde. $\mathrm{O}$ autor afirma a importância da criação de estratégias de comunicação com essa população para melhorar a atenção prestada.

Segundo os profissionais, a visão do modelo assistencial de atenção e da busca apenas de atendimento de emergência, sem a valorização da promoção da saúde e da prevenção de doenças influencia a adequada utilização do serviço de saúde e sobrecarrega o sistema: [...] para eles, o que importa é a resolubilidade. "Lá (UPA) eu consigo, lá eles me atendeme fazem exame na hora" (profissional de nível superior 2, reproduzindo a fala de um usuário). Pucciniet al. (2012) questionaram em seu estudo sobre acesso com profissionais da saúde sobre a abrangência das funções da atenção básica e evidenciaram diferentes posições. Parte dos entrevistados afirmaram que a atenção básica deveria estar voltada para a promoção e a prevenção, e descartaram que caberia à UBS atender a demanda espontânea. Em decorrência das diversas concepções, ficou evidente que as orientações obtidas pelos usuários nos serviços básicos de saúde são confusas e contraditórias, predominando uma orientação restritiva à utilização das unidades básicas. Essa recusa ao atendimento da demanda não agendada refletiria em restrições no acesso às unidades básicas de saúde.

De acordo com os profissionais de saúde, a elevada demanda teria relação direta com a condição precária de vida da população local, que utiliza o serviço de saúde como uma "válvula de escape" de sua situação de vida: eu acho que é o perfil da população, que tem o nível socioeconômico mais baixo, eles procuram muito o serviço de saúde (gestor 3 ). Esse uso indevido levaria ao prejuízo dos demais usuários, "inchando" a agenda com consultas "desnecessárias”. Mas o que são problemas de saúde? É provável que os serviços de saúde não sejam capazes de resolver grande parte dos problemas dessa população, mas não é possível excluí-los do contexto de indivíduo que procura o atendimento. Muitas vezes, esses problemas estão diretamente relacionados ao processo de saúde-doença do usuário. Não se pode dissociar saúde de alimentação, moradia, segurança, relações psicossociais, entre outros fatores. Entretanto, podem-se criar espaços de acolhimento dessas demandas mais subjetivas e intersetoriais.

Autores que tratam da questão do acesso (Mendo-
za-Sassi; Beria, 2001; Almeida et al., 20oo) discutem que a maior utilização de serviços de saúde pela população de menor nível socioeconômico estaria associada à maior morbidade desse grupo. Quando se estratifica grupos de níveis socioeconômicos superiores e inferiores, de acordo com o grau de morbidade, tem-se que os grupos mais pobres utilizam menos os serviços que os grupos mais ricos. Rosa Filho et al. (2008) falam que o aumento da procura é devido, também, à insatisfação do usuário com a qualidade do serviço ofertado.

Para Taddeo et al. (2012), o usuário deve ser abordado enquanto cidadão e protagonista. 0 autocuidado evidencia-se de forma relacional, sendo que paciente e profissional dependem um do outro para realizar o cuidado. 0 conhecimento e o acesso às informações são imprescindíveis para a realização de escolhas e empoderamento para ação.

Em contrapartida, a subutilização do serviço de saúde também emergiu na fala dos profissionais associada ao preconceito dos usuários com o SUS, que deixariam de procurar pelos serviços já imaginando que não seriam bem atendidos: então eles às vezes deixam de vir procurar o serviço porque já tem lá um preconceito de que irá demorar [...] (profissional de nível superior 2).

Thiede e McIntyre (2008), quando discorrem sobre aceitabilidade, deixam clara a importância do grau de segurança que os serviços têm perante a população, se eles são culturalmente aceitos pela comunidade. Goddard e Smith (2001) destacam que os conhecimentos sobre disponibilidade e eficácia de serviços de saúde entre os indivíduos e suas percepções dos benefícios da assistência oferecida são fatores que podem influenciar o acesso aos serviços.

Sobre esse aspecto, vale ressaltar que, neste estudo, apenas 26,3\% (10) usuários consideraram o serviço de saúde bom (Tabela 3). Houve relatos de $36,9 \%$ (14) usuários sobre a necessidade de ir duas ou mais vezes ao serviço de saúde para resolver seu problema, sendo que $55,3 \%$ (21) disseram procurar outro local quando não tinham seu problema resolvido.

Os serviços da APS disponibilizados na UBR estudada e em Unidades de Saúde foram citados por 71,1\% (27) usuários como os locais rotineiramente procurados para atendimento das necessidades de saúde. É interessante notar, no entanto, que o motivo 
por essa procura, relatado por 53,0\% (18/34) usuários, esteve relacionado à acessibilidade geográfica, ou seja, à proximidade com a residência (Tabela 3). Motivos relacionados à qualidade do serviço prestado, como satisfação, eficiência e boa relação com os profissionais de saúde não foram citados. No trabalho de Oliveira et al. (2012), sobre a acessibilidade à atenção básica em um distrito sanitário de Salvador, a principal razão para a procura da unidade de referência foi a proximidade geográfica (45,7\%), entretanto, diferentemente do presente trabalho, a qualidade do atendimento $(28,1 \%)$ foi a segunda causa citada.

Acrescenta-se a essa discussão, a análise dos motivos citados para a utilização de serviços externos ao bairro, sendo os principais a ausência de vagas para consulta ou de médicos no serviço do bairro $(34,2 \%)$ e percepção de doença grave $(21,1 \%)$, que pode demonstrar uma falta de confiança no serviço, fator importante para o acesso (Tabela 3). As principais dificuldades de utilização do serviço de saúde do bairro relatadas pelos usuários foram: falta de médicos $(36,8 \%)$ e demora da marcação de consultas (31,6\%) (Tabela 3).

No que diz respeito à relação do usuário com o profissional de saúde, foi relatado por estes que há uma grande dificuldade no estabelecimento de vínculo, devido, principalmente, à grande rotatividade de profissionais. Nota-se que, em seus depoimentos, um gestor e um profissional assistencial de nível superior abordaram visões distintas do problema. 0 gestor relacionou o problema da rotatividade à falta de comprometimento dos profissionais com os serviços; já o profissional da assistência associou a questão à falta de políticas de incentivo aos trabalhadores da saúde: [...] o profissional médico que vem para o município, acho que pra qualquer serviço público, ele não é compromissado. [...] $\varepsilon$ o serviço público e a atenção primária realmente não é o foco dele de vida profissional. Ali é um degrau apenas (gestor 3); [...] os nossos gestores têm que olhar com mais carinho para os recursos humanos, vamos valorizar os recursos humanos, vamos fixar os nossos profissionais nas unidades [...] (profissional de nível superior 4).

Pelo questionário aplicado aos usuários, foi possível perceber a demanda pela longitudinalidade (o vínculo do usuário com a unidade e/ou com o profissional), expressa na procura pelo mesmo serviço de saúde/médico, relatada por $94,7 \%$ dos entrevistados. 0 fato de apenas 50\% dos usuários relatarem que eram sempre atendidos pelos mesmos profissionais, quando procuravam o serviço de saúde, reforça a questão da rotatividade de profissionais abordada nas entrevistas com os gestores e profissionais de saúde.

Observa-se, assim, uma relação de pouca confiança também entre gestores do sistema e profissionais de saúde e, embora esta problemática não seja habitualmente citada como um fator determinante para o acesso impacta, mesmo que indiretamente, como se pôde notar, no acesso da população aos serviços, seja pela pequena fixação de profissionais, seja pela falta de eficiência no atendimento, que interferem na integralidade e na qualidade do acesso.

$\mathrm{Na}$ abordagem da relação entre o usuário e o profissional de saúde, Thiede e McIntyre (2008) discutem a importância da interação entre usuários e prestadores para a melhoria do acesso, devendo a informação ser foco de políticas públicas de saúde. Os autores abordam o papel da educação no acesso e como este pode ser determinado pela posição social do indivíduo, destacam que o processo educativo deve ser participativo e enfatizam que a informação e o empoderamento são pré-requisitos para o acesso.

\section{Considerações finais}

Este estudo, de maneira geral, corrobora a compreensão multidimensional do acesso aos serviços de saúde (Thiede; McIntyre, 2008; Donabedian, 1973, 2003; Giovanella; Fleury, 1996) e sua relação com as condições de saúde e vida da população.

Embora seja importante mencionar as limitações do estudo que não envolveu os não usuários e a amostra não ter sido aleatória, os resultados evidenciaram fatores determinantes para o acesso do grupo estudado, incluindo: a aceitabilidade do sistema de saúde pelos usuários, a relação deles com os profissionais de saúde, a adequação do número de profissionais para a prestação de serviços, a atenção secundária/referência, o financiamento do sistema de saúde e a estigmatização do município, apontando que, embora a dimensão "operacional" 
seja determinante para o acesso do grupo estudado, a dimensão "relacional" tem um papel fundamental.

Nesse sentido, investimentos e esforços de gestores e profissionais de saúde, sobretudo aqueles envolvidos diretamente com a UBR estudada, devem ser direcionados a essas duas dimensões. Essa possibilidade de identificar, sob a ótica de usuários e profissionais, áreas prioritárias de atuação para melhorar o acesso, de uma parcela da população de um município com elevados índices de vulnerabilidade econômica e social, configura a principal contribuição deste estudo.

\section{Referências}

ALMEIDA, C. et al. Health sector reform in Brazil: a case study of inequity. International Journal of Health Services, Baltimore, v. 30, n. 1, p. 129-162, 2000.

ANDERSEN, R. M. Revisiting the behavioral model and access to medical care: does it matter? Journal of Health and Social Behavior, Los Angeles, v. 36, n. 1, p. 1-10, 1995.

ANDRULIS, D. P. Community, service, and policy strategies to improve health care access in the changing urban environment. American Journal of Public Health, Birmingham, v. 9o, n. 6, p. 858-862, 2000.

BARDIN, L. Análise de conteúdo. Lisboa: Edições 70, 2010.

BRASIL. Presidência da República. Emenda Constitucional $n^{0} 29$, de 13 de setembro de 2000. Altera os artigos 34, 35, 156, 160, 167 e 198 da Constituição Federal e acrescenta artigo ao Ato das Disposições Constitucionais Transitórias, para assegurar os recursos mínimos para o financiamento das ações e serviços públicos de saúde. Diário Oficial [da] República Federativa do Brasil, Brasília, DF, 13 set. 20oo. Disponível em: <http://www.planalto.gov.br/ccivil_03/ constituicao/Emendas/Emc/emc29.htm>. Acesso em: 7 dez. 2011.

CANGUILHEM, G. O normal e o patológico. 6. ed. São Paulo: Forense Universitária, 2000.
CUNHA, A. B. O.; VIEIRA-DA-SILVA, L. M.

Acessibilidade aos serviços de saúde em um município do Estado da Bahia, Brasil, em gestão plena do sistema. Cadernos de Saúde Pública, Rio de Janeiro, v. 26, n. 4, p. 725-737, 2010.

DONABEDIAN, A. Aspects of medical care administration. Cambridge: Harvard University, 1973.

DONABEDIAN, A. An introduction to quality assurance in health care. New York: Oxford University, 2003.

GIOVANELLA, L.; FLEURY, S. Universalidade da atenção à saúde: acesso como categoria de análise. In: EIBENSCHUTZ, C. (Org.). Política de saúde: o público e o privado. Rio de Janeiro: Fiocruz, 1996. p. 177-198.

GODDARD, M.; SMITH, P. Equity of access to health care services: theory and evidence from the UK. Social Science \& Medicine, Boston, v. 53, n. 9, p. 1149-1162, 2001.

JESUS, W. L. A.; ASSIS, M. M. A. Revisão sistemática sobre o conceito de acesso nos serviços de saúde: contribuições do planejamento. Ciência \& Saúde Coletiva, Rio de Janeiro, v. 15, n. 1, p. 161-170, 2010.

MENDOZA-SASSI, R.; BERIA, J. U. Utilización de los servicios de salud: una revisión sistemática sobre los factores relacionados. Cadernos de Saúde Pública, Rio de Janeiro, v. 17, n. 4, p. 819832, 2001.

MINAYO, M. C. de S.; DESLANDES, S. F.; GOMES, R. Pesquisa social: teoria, método e criatividade. 29. ed. Petrópolis: Vozes, 2010.

MOIMAZ, S. A. S. et al. Satisfação e percepção do usuário do SUS sobre o serviço público de saúde. Physis: Revista de Saúde Coletiva, Rio Janeiro, v. 20, n. 4, p. 1419-1440, 2010.

OBRIST, B. et al. Access to health care in contexts of livelihood insecurity: a framework for analysis and action. PLOS Medicine, Cambridge, v. 4, n. 10, p. 1584-1588, 2007.

OJIMA, R. et al. O estigma de morar longe da cidade: repensando o consenso sobre as "cidadesdormitório" no Brasil. Cadernos Metrópole, São Paulo, v. 12, n. 24, p. 395-415, 2010. 
OLIVEIRA, L. S. et al. Acessibilidade à atenção básica em um distrito sanitário de Salvador. Ciência \& Saúde Coletiva, Rio de Janeiro, v. 17, n. 11, p. 3047-3056, 2012.

PENA, P. F. A. et al. Cuidado ao paciente com doença renal crônica no nível primário: pensando a integralidade e o matriciamento. Ciência $\&$ Saúde Coletiva, Rio de Janeiro, v. 17, n. 11, p. 31353144, 2012.

PENCHANSKY, D. B. A.; THOMAS, J. W. The concept of access: definition and relationship to consumer satisfaction. Medical Care, West Chester, v. 19, n. 2, p. 127-140, 1981.

PINHEIRO, R. S. et al. Gênero, morbidade, acesso e utilização de serviços de saúde no Brasil. Ciência \& Saúde Coletiva, Rio de Janeiro, v. 7, n. 4, p. 687707, 2002.

PUCCINI, P. T. et al. Concepção de profissionais de saúde sobre o papel das unidades básicas nas redes de atenção do SUS/Brasil. Ciência \& Saúde Coletiva, Rio de Janeiro, v. 17, n. 11, p. 2941-2952, 2012.

RIPSA - REDE INTERAGENCIAL DE INFORMAÇÕES PARA A SAÚDE. Gasto com ações e serviços públicos de saúde per capita - E.6.2 2011. Brasília, DF, 2011. Disponível em: <http:// fichas.ripsa.org.br/2011/E-6-2/?l=pt_BR>. Acesso em: 7 dez. 2011.
ROSA FILHO, L. A. et al. Fatores associados à continuidade interpessoal na atenção à saúde: estudo de base populacional. Cadernos de Saúde Pública, Rio de Janeiro, v. 24, n. 4, p. 915-925, 2008.

SANTOS, L.; ANDRADE, L. O. M. Acesso às ações e serviços de saúde: uma visão polissêmica. Ciência \& Saúde Coletiva, Rio de Janeiro, v. 17, n. 11, p. 2876-2878, 2012.

TADDEO, P. S. et al. Acesso, prática educativa e empoderamento de pacientes com doenças crônicas. Ciência \& Saúde Coletiva, Rio de Janeiro, v. 17, n. 11, p. 2923-2930, 2012.

THIEDE, M.; McINTYRE, D. Information, communication, and equitable access to health care. Cadernos de Saúde Pública, Rio de Janeiro, v. 24, n. 5, p. 1168-1173, 2008.

TRAVASSOS, C.; MARTINS, M. Uma revisão sobre os conceitos de acesso e utilização de serviços de saúde. Cadernos de Saúde Pública, Rio de Janeiro, v. 20, n. 2, p. 190-198, 2004.

TRAVASSOS, C.; VIACAVA, F. Acesso e uso de serviços de saúde em idosos residentes em áreas rurais, Brasil, 1998 e 2003. Cadernos de Saúde Pública, Rio de Janeiro, v. 23, n. 10, p. 2490-2502, 2007.

\section{Contribuição dos autores}

Viegas, Carmo e Luz colaboraram na concepção do estudo, análise dos resultados e redação do artigo.

Recebido: 06/08/2013

Reapresentado: 13/02/2014

Aprovado: 10/03/2014 